\title{
Factores predictores perioperatorios de complicaciones de la colecistectomía por laparoscopia
}

\author{
Perioperative complications predictive risk factors in laparoscopic \\ cholecystectomy
}

\author{
Guillermo Eduardo Aldana1, Luis Eduardo Martínez², Manuel Augusto Hosman³, Diego \\ Alejandro Ardila ${ }^{3}$, Iván Fernando Mariño ${ }^{3}$, Michael Ricardo Sagra ${ }^{3}$, Luisa María Montoya ${ }^{4}$ \\ Cirujano adscrito, Hospital Infantil Universitario San José, Bogotá, Vicepresidente Asociación Colombiana de Cirugía Hepatobiliar, \\ Bogotá, D.C., Colombia \\ 2 Médico, cirujano; instructor asistente, Departamento de Cirugía General, Fundación Universitaria de Ciencias de la Salud, Hospital \\ de San José, Bogotá, D.C., Colombia \\ 3 Médico, Cirujano General, Hospital San José, Bogotá, D.C., Colombia \\ 4 Médica, instructor asistente, División de Investigaciones, Fundación Universitaria de Ciencias de la Salud, Bogotá, D.C., Colombia \\ Trabajo presentado en el concurso del residente quirúrgico, 43 Congreso Nacional "Avances en Cirugía", 2017, Medellín, Colombia
}

\section{Resumen}

Introducción. Los factores de riesgo para la conversión a laparotomía y las complicaciones de la colecistectomía laparoscópica se han estudiado, pero no se conocen modelos actuales de predicción para estos resultados.

Objetivo. Desarrollar un modelo de predicción para las complicaciones de la colecistectomía laparoscópica.

Pacientes y métodos. Se llevó a cabo un estudio analítico retrospectivo que incluyó I.234 pacientes con colelitiasis sometidos a colecistectomía laparoscópica, en un periodo de I8 meses en un hospital de IV nivel de Colombia. Se hizo el análisis multivariado por medio de regresión logística, usando el procedimiento backward para selección de variables, buscando determinar la probabilidad en un punto compuesto de complicación (presencia de, al menos, una complicación: lesión de vía biliar, colección intraabdominal o sangrado). Se elaboró una curva ROC para determinar la capacidad predictiva del modelo y el análisis de datos se hizo en Stata $13^{\mathrm{TM}}$.

Resultados. Los pacientes incluidos se clasificaron en cohortes de derivación (926) y de validación (308), y se encontró que el 69,2 \% eran mujeres; la edad mediana fue de 48 años (RIC=34-60) y, la conversión, de 4,3\%; hubo colección intraabdominal en 2,6 \% y complicaciones en 4,7\%, y la mortalidad global fue de 0,3\%. La edad, la diabetes mellitus, la enfermedad renal crónica, la coledocolitiasis y el síndrome de Mirizzi se identificaron como predictores de alguna complicación. La capacidad predictiva del modelo fue de $58 \%$.

Conclusión. La probabilidad de alguna complicación perioperatoria de la colecistectomía por laparoscopia depende de la edad, la diabetes mellitus, la enfermedad renal crónica, la coledocolitiasis y el síndrome de Mirizzi. Los factores de riesgo y las complicaciones descritos aquí, pueden guiar nuevas investigaciones y proporcionar evaluación de riesgos específicos.

Palabras clave: colelitiasis; colecistectomía laparoscópica; complicaciones intraoperatorias; complicaciones posoperatorias; medición de riesgo; probabilidad.

Fecha de recibido: 26/05/2017 - Fecha aceptación: 16/02/2018

Correspondencia: Guillermo Eduardo Aldana, MD, Calle 10 Nº18-75, Hospital de San José, Servicio de Cirugía General, Bogotá, D. C., Colombia / Teléfono: (314) 356-9931

Correo electrónico: aesculap12@gmail.com

Citar como: Aldana GE, Martínez LE, Hosman MA, Ardila DA, Mariño IF, Sagra MR, et al. Factores predictores perioperatorios de complicaciones de la colecistectomía por laparoscopia. Rev Colomb Cir. 2018;33:162-72. https://doi.org/10.30944/20117582.58 


\begin{abstract}
Introduction. Risk factors associated with conversion from laparoscopic cholecystectomy to open surgery and its complications are well known. However, there are currently no prediction models for such outcomes. The objective of tis work was to devolop a prediction model for laparoscopic cholecystectomy complications.

Materials and methods. This is a retrospective analytical study that included I,234 patients who underwent laparoscopic cholecystectomy in an I8 months period at a fourth level of care hospital in Bogota, Colombia. A multivariable logistic regression analysis using backward procedure was performed to for the selection of variables, in order to determine the likelihood of a combined endpoint complication (presence of at least one of the complications: bile ducts injury, haemorrhage, organ/space surgical site infection). A ROC curve was performed to determine the predictive ability of the model; information analysis was performed in I3 STATA.

Results. Patients were classified in a derivation (926) and a validation cohort (308). It was found that $69.2 \%$ were female, median age 48 years (IQR 34-60), conversion rate $4.3 \%$, organ/space surgical site infections $2.6 \%$, combined end point complication $4.7 \%$, and global mortality rate $0.3 \%$. Diabetes mellitus (DM), chronic kidney disease (CKD), choledocholitiasis and Mirizzi's syndrome were found as predictors of the occurrence of complications. The model was validated in the validation cohort, obtaining an area under the ROC curve of $58 \%$.

Discussion. The likelihood of major complication in laparoscopic cholecystectomy depends on age, DM, CKD, choledocholitiasis, and Mirizzi's syndrome. Risk Factors and complications described here can guide a new research avenue and provide the evaluation of specific risks.
\end{abstract}

Keywords: cholelithiasis; cholecystectomy, laparoscopic; intraoperative complications; postoperative complications; risk assessment; probability

\section{Introducción}

De las enfermedades gastrointestinales, la biliar es la segunda causa más común de hospitalización en los países desarrollados ${ }^{\mathrm{I}}$. Se estima que entre el Io y el I5 \% de la población norteamericana tiene colelitiasis, de la cual, del io al i8 \% cursa con síntomas y el $7 \%$ requiere intervención quirúrgica ${ }^{2}$.

La prevalencia mundial de la colelitiasis varía de acuerdo con diferencias socioeconómicas, étnicas y raciales, incluso entre países del mismo continente, siendo mayor entre poblaciones indígenas, incluso en Estados Unidos, y encontrándose prevalencias muy bajas en los países africanos. En Latinoamérica, la prevalencia es alta en general; Chile es el país con la prevalencia más alta del mundo: $44 \%$ de las mujeres y $25 \%$ de las mujeres mayores de 20 años ${ }^{3}$. En Colombia, no se cuenta con estudios poblacionales de prevalencia nacional de colelitiasis, sin embargo, en un estudio en el Hospital Universitario Fundación Santa Fe de Bogotá en 200I, de Botero, et al., se encontró una prevalencia de $8,6 \%$ en hombres ${ }^{3}$.

La incidencia de la colelitiasis aumenta con la edad a razón de I a $3 \%$ al año ${ }^{4}$ y cursa con mayor frecuencia en mujeres con una razón de mujer a hombre de $4 \mathrm{a} \mathrm{I}^{3}$.

La colecistitis aguda representa del I al $3 \%$ de los casos de hospitalización, como principal complicación de la colelitiasis ${ }^{2,5}$. La gravedad del cuadro agudo propicia, principalmente, una mayor dificultad técnica ${ }^{5,6}$ y otras complicaciones de la colelitiasis, entre las que se cuentan la pancreatitis, la coledocolitiasis y el íleo biliar, aunque con menor incidencia que la colecistitis (7); también, desencadenan mayor estancia hospitalaria, secuelas y morbilidades, lo cual genera mayores costos sociales y económicos.

Los factores de riesgo de las complicaciones en la colecistectomía por laparoscopia, han sido bien identificados ${ }^{8,9}$. Generalmente, están relacionados con la edad, la cirugía urgente, la obesidad y las enfermedades concomitantes ${ }^{9}$, además de los factores bioquímicos y los relacionados con la cirugía, que generan dificultad para identificar las estructuras del triángulo de Calot ${ }^{10}$.

La colecistitis aguda se comporta también como un factor de riesgo de complicación en la colecistectomía por laparoscopia ${ }^{2}$ y se mantiene 
como factor predictivo para la conversión a cirugía abierta, con una asociación significativa (odds ratio, $\left.\mathrm{OR}=5,6 ; \mathrm{IC}_{95 \%} 2,09-\mathrm{I} 5, \mathrm{I} 5\right)^{\mathrm{II}-\mathrm{I} 3}$.

Otras complicaciones, como la lesión de la vía biliar que ostenta una incidencia de o,I a o,6 \%, las lesiones vasculares (0,9 por I.00o), las lesiones intestinales (I,8 por I.OoO) ${ }^{14} \mathrm{y}$ las colecciones intraabdominales, pueden estar asociadas con factores clínicos, como edad mayor de 65 años $\left(\mathrm{OR}=3,025 ; \mathrm{IC}_{95} \% \mathrm{I}, \mathrm{O} 7-8,5 \mathrm{O}\right){ }^{\mathrm{II}}$, el sexo masculino $\left(\mathrm{OR}=\mathrm{I}, \mathrm{I} 4 ; \mathrm{IC}_{95} \% \mathrm{I}, \mathrm{IO}-\mathrm{I}, \mathrm{I9}\right){ }^{15}$, la cirugía urgente $\left(\mathrm{OR}=\mathrm{I}, 69 ; \mathrm{IC}_{95 \%} \mathrm{I}, 59-\mathrm{I}, 8 \mathrm{I}\right){ }^{15} \mathrm{y}$ comorbilidades como la diabetes mellitus ( $\left.\mathrm{OR}=3,85 ; \mathrm{IC}_{95} \% \mathrm{I}, 50-9,84\right)$, y hallazgos quirúrgicos como el plastrón vesicular $\left(\mathrm{OR}=23,5 ; \mathrm{IC}_{95 \%} 8,89-62,46\right)^{16}$.

A pesar de que estas asociaciones están descritas actualmente como 'evidencias', la planeación de medidas de prevención previas al acto quirúrgico para evitar las complicaciones mencionadas, depende de la identificación de estos factores como predictores en la población de interés.

El objetivo de este estudio fue ajustar un modelo predictivo de las variables clínicas, bioquímicas y operatorias para un punto de complicación compuesto (definido como la presencia de, por lo menos, una de las siguientes complicaciones: lesión de la vía biliar, sangrado o colección intraabdominal), en pacientes sometidos a colecistectomía por laparoscopia.

\section{Métodos}

Los datos presentados son el resultado de un estudio analítico retrospectivo de tipo cohorte, que incluyó a todos los pacientes con colelitiasis sometidos a colecistectomía por laparoscopia durante el periodo comprendido entre agosto de 2013 y enero de 2015, registrados en el sistema del Servicio de Cirugía General en un hospital universitario de IV nivel considerado como centro de referencia en el manejo de la enfermedad biliar en Bogotá.

La información fue recolectada a partir de las historias clínicas digitales del hospital, y registrada en una base de datos diseñada para tal fin. Se excluyeron del estudio los pacientes sometidos a colecistectomía abierta u operados en una institución diferente, así como aquellos intervenidos por cirugía pediátrica.

Como resultado principal se evaluó un punto compuesto de complicación, con alguna de las siguientes variables: lesión de vía biliar, sangrado o colección intraabdominal.

Se consideró 'conversión' a aquella cirugía iniciada por vía laparoscópica y continuada como una intervención abierta; aunque la conversión no se considera actualmente como una complicación de la cirugía laparoscópica, se incluyó para fines del estudio, teniendo en cuenta que impacta la morbilidad posoperatoria.

El sangrado se consideró como significativo cuando requirió manejo quirúrgico o transfusión en el periodo posoperatorio inmediato. La lesión intestinal y vascular mayor se tuvo en cuenta cuando se requirió algún tipo de intervención para su control.

Se consideró infección del sitio operatorio de tipo colección intraabdominal, toda colección intraabdominal que requirió intervención percutánea o quirúrgica para su drenaje.

Las lesiones de la vía biliar se incluyeron como complicaciones, independientemente de su grado de complejidad.

Las complicaciones médicas, como la tromboembolia pulmonar, la trombosis venosa profunda, el infarto agudo del miocardio y otras, entre las que se tuvieron en cuenta la neumonía, las atelectasias, el derrame pleural, la falla renal aguda, la descompensación de la falla cardíaca, la descompensación de la enfermedad pulmonar obstructiva crónica (EPOC) y la trombosis de la porta, también fueron evaluadas.

Como antecedentes quirúrgicos, se tuvo en cuenta únicamente el antecedente quirúrgico abdominal. El tiempo quirúrgico considerado como factor de riesgo fue aquel mayor de 72 horas desde el ingreso, siguiendo lo sugerido en la guía de Tokio para el manejo de la colecistitis aguda - TGI3 ${ }^{9}$; como técnicas de inserción quirúrgica, solo se consideraron la abierta y el trocar óptico, debido a que en la institución de estudio solo se practica este tipo de procedimientos. 
El análisis estadístico se hizo con el programa Stata $\mathrm{I}^{\mathrm{TM}}$, calculando las medidas de tendencia central y de dispersión para las variables cuantitativas. La cohorte total de pacientes se dividió en dos; una cohorte de derivación (75\%) y una cohorte de validación ( $25 \%$ ), las cuales presentaron un comportamiento homogéneo en cuanto a las características clínicas y demográficas.

Para evaluar la relación entre las complicaciones y los factores de riesgo propuestos en la literatura, se hizo un análisis multivariado en la cohorte de derivación por medio de una regresión logística para el punto de complicación compuesto. Para la realización de este modelo, se planteó obtener Io resultados por cada variable, y se esperaba contar con un mínimo de cinco variables en el modelo, por lo que fue necesario recolectar 50 complicaciones como mínimo.

El modelo quedó determinado por las variables independientes que presentaron una significación de 0,2 en el análisis bivariado con el resultado, el cual fue valorado mediante la prueba de ji al cuadrado para las variables cualitativas y con la prueba de Mann-Whitney para las variables cuantitativas, dada la falta de normalidad de los datos, la cual fue probada mediante la prueba de Shapiro-Wilk.

Para llegar al modelo final, se empleó el procedimiento backward para la selección de las variables independientes, considerando un valor de p de 0,05 como significativo desde el punto de vista estadístico. Los supuestos del modelo se evaluaron por medio de gráficos de residuales; además, se evaluó la colinealidad mediante el valor de inflación de la varianza. El modelo fue validado en la cohorte de validación mediante la construcción de una curva ROC, con el fin de determinar la capacidad de clasificación del modelo. La bondad de ajuste se evaluó mediante los criterios de información de Akaike y bayesiano.

El estudio fue aprobado por el Comité de Investigaciones de la Fundación Universitaria de Ciencias de la Salud y se clasificó como estudio sin riesgo según la Resolución oo8430 de 1993 del Ministerio de Salud de Colombia; se acataron todas las consideraciones éticas.

\section{Resultados}

Durante los I8 meses de recolección de datos, se practicaron I.293 colecistectomías, de las cuales I.234 cumplieron criterios de elegibilidad incluidos en el presente análisis. La mediana de la edad de los pacientes incluidos fue de 48 años (rango intercuartílico, RIC, 34 a 60) y el 69,2 \% correspondió al sexo femenino.

Con respecto a los antecedentes clínicos, la comorbilidad más frecuente fue la hipertensión arterial sistémica (22,6\%), seguida por la diabetes mellitus de tipo 2 (7,8\%). Hubo antecedentes quirúrgicos abdominales en el 44,I \% de los pacientes. En todas las complicaciones fue más frecuente el ingreso a la cavidad abdominal mediante técnica abierta, excepto entre los pacientes que murieron ${ }^{4}$, dos de los cuales tuvieron ingreso mediante trocar óptico, sin ninguna relación directa.

En el perfil hepático, que incluyó bilirrubinas total, directa e indirecta, fosfatasa alcalina, amilasemia, aspartato aminotransferasa (AST), alanina aminotransferasa (ALT) y un marcador de reacción inflamatoria, como los leucocitos, se encontraron medianas dentro de los rangos normales (tabla I).

La prevalencia de la coledocolitiasis fue de II,6 \% y todos estos pacientes fueron sometidos a colangiopancreatografía retrógrada endoscópica (CPRE). El diámetro del colédoco fue anormal ( $>6 \mathrm{~mm}$ ) en $8,4 \%$ de los pacientes, y en $26,2 \%$ no se encontró información respecto al diámetro del colédoco.

En la mayoría de las cirugías se ingresó a la cavidad abdominal por medio de una técnica abierta $(72,4 \%)$ y, con menor frecuencia, se intervino con tres o menos puertos de laparoscopia $(5,4 \%)$. La mayoría de los pacientes fueron operados de forma urgente $(63,9 \%) \mathrm{y}$, entre los llevados a colecistectomía programada, el ingreso más usual fue por medio de trocar óptico $(58,7 \%)$ y fue más frecuente la cirugía con tres o menos puertos, en comparación con las cirugías urgentes (I,9 Vs. II,6 \%) (tabla 2).

Hubo algún tipo de hallazgo quirúrgico relacionado con una complicación de la colelitiasis, 
Tabla 1. Características basales de la población $(n=1234)$

\begin{tabular}{lc}
\hline & $\mathbf{n}(\%)$ \\
\hline Características sociodemográficas & \\
Edad (años), mediana (RIQ) años & $48(34-60)$ \\
Sexo: mujeres & $854(69,2)$ \\
Antecedentes ( $\mathrm{n}=1.229)$ & \\
Hipertensión arterial sistémica & $278(22,6)$ \\
EPOC & $19(1,6)$ \\
Diabetes mellitus & $96(7,8)$ \\
Enfermedad renal crónica & $20(1,6)$ \\
Inmunosupresión & $34(2,8)$ \\
Antecedente quirúrgico abdominal & $543(44,2)$ \\
Características bioquímicas & \\
Bilirrubina total (mg/dl): mediana (RIQ) (n=990) & \\
Fosfatasa alcalina (mg/dl): mediana (RIQ) (n=976) & $0.9(0.6-1.8)$ \\
Amilasa (mg/dl): mediana (RIQ) (n=821) & $104,5(80-161)$ \\
Aspartato aminotransferasa, AST (mg/dl): mediana (RIQ) (n=991) & $61(47-85)$ \\
Alanino aminotransferasa, ALT(mg/dl): mediana (RIQ) (n=990) & $31(22-107)$ \\
Leucocitos: mediana (RIQ) (n=930) & $43(28-152)$ \\
Características clínicas & $9.400(7.200-12.800)$ \\
CPRE preoperatoria & \\
Colédoco: >6 mm según ecografía (n=911) & $143(11,6)$ \\
\hline RIQ: rango intercuartílico; EPOC: enfermedad pulmonar obstructiva crónica; CPRE: colangiopan- \\
creatografía retrógrada endoscópica
\end{tabular}

en 72,9\% de los pacientes: con mayor frecuencia en la cirugía urgente $(99,8 \%)$ que en la cirugía programada (25,2 \%); el principal hallazgo en todos los pacientes fue la colecistitis, presente en el 43,5\%. La mediana de estancia hospitalaria fue de dos días ( $\mathrm{RIC}=\mathrm{I}-4)$, la cual fue mayor en la cirugía urgente (mediana $=3 ; \mathrm{RIC}=2-5$ ) que en la cirugía programada (mediana $=\mathrm{I} ; \mathrm{RIC}=\mathrm{I}-\mathrm{I}$ ). En total, se encontraron $\mathrm{I} 7$ (I,4 \%) complicaciones médicas, 13 con cirugía urgente y 4 con cirugía programada (tabla 2).

La conversión a cirugía abierta tuvo una incidencia de $4,3 \%$, mayor en las cirugías urgentes $(6, \mathrm{I} \%)$ que en las programadas $(\mathrm{I}, \mathrm{I} \%)$; de la misma manera, las lesiones de la vía biliar, la lesión vascular y el sangrado, presentaron mayor incidencia en cirugías de urgencia y fueron más frecuentes en hombres.
Se presentaron colecciones intraabdominales que requirieron algún tipo de intervención quirúrgica o percutánea, en 32 pacientes $(2,6 \%)$, sin mayores diferencias entre las cirugías urgentes $(2,8 \%)$ y las programadas $(2,3 \%)$.

La mortalidad global fue de $0,3 \%$, con tres $(0,4 \%)$ casos entre las cirugías urgentes y I $(0,2 \%)$ caso entre las programadas (tabla 3 ).

La única complicación que fue más frecuente en las cirugías programadas fue la lesión intestinal, con tres de cinco casos en total.

Como resultado principal se evaluó un punto compuesto de complicación con alguna de las siguientes variables: lesión de vía biliar, sangrado o colección intraabdominal, encontrándose una incidencia de 4,7\% del total de la población estudiada. Al hacer el análisis bivariado con el resultado, se encontró que las variables sexo, edad, 
Tabla 2. Características quirúrgicas y resultados clínicos según tipo de cirugía

\begin{tabular}{lccc}
\hline & $\begin{array}{c}\text { Urgente } \\
\mathbf{n = 7 8 9}(\mathbf{6 3 , 9}) \\
\mathbf{n}(\%)\end{array}$ & $\begin{array}{c}\text { Programada } \\
\mathbf{n = 4 4 5}(\mathbf{3 6 , 1}) \\
\mathbf{n}(\%)\end{array}$ & $\begin{array}{c}\text { Total } \\
\mathbf{n = 1 2 3 4} \\
\mathbf{n}(\%)\end{array}$ \\
\hline $\begin{array}{l}\text { Tiempo desde ingreso hasta la cirugía >72 horas } \\
\text { Técnica de inserción del trocar }\end{array}$ & $225(28,7)$ & $2(0,5)$ & $227(18,5)$ \\
Abierta & $710(90,0)$ & $184(41,4)$ & $894(72,5)$ \\
Óptico & $79(10,0)$ & $261(58,7)$ & $340(27,6)$ \\
Puertos & & & \\
Uno & 0 & $9(2,0)$ & $9(0,73)$ \\
Dos & $3(0,4)$ & $18(4,0)$ & $21(1,7)$ \\
Tres & $12(1,5)$ & $25(5,6)$ & $37(3,0)$ \\
Cuatro & $774(98,1)$ & $393(88,3)$ & $1167(94,6)$ \\
Hallazgos quirúrgicos & & & \\
Hidrocolecisto & $22(2,8)$ & $7(1,6)$ & $29(2,4)$ \\
Piocolecisto & $104(13,2)$ & $8(1,8)$ & $112(9,1)$ \\
Plastrón vesicular & $161(20,4)$ & $17(3,8)$ & $178(14,4)$ \\
Colecistitis & $466(59,1)$ & $71(16,0)$ & $537(43,5)$ \\
Síndrome de Mirizzi & $34(4,3)$ & $9(2,0)$ & $43(3,5)$ \\
Estancia hospitalaria mediana (RIQ) días & $3(2-5)$ & $1(1-1)$ & $2(1-4)$ \\
Complicaciones médicas & & & \\
Tromboembolia pulmonar & $1(0,1)$ & $1(0,2)$ & $2(0,2)$ \\
Trombosis venosa profunda & 0 & $1(0,2)$ & $1(0,1)$ \\
Infarto agudo de miocardio & $1(0,1)$ & 0 & $1(0,1)$ \\
Otras & $11(1,4)$ & $2(0,5)$ & $13(1,1)$ \\
\hline
\end{tabular}

RIQ: rango intercuartílico

hipertensión arterial sistémica, diabetes mellitus, enfermedad renal crónica, fosfatasa alcalina, diagnóstico de coledocolitiasis, antecedente de colangiopancreatografía retrógrada endoscópica, tiempo quirúrgico, técnica de inserción, piocolecisto, síndrome de Mirizzi, plastrón, conversión, lesión intestinal y estancia hospitalaria presentaron valores de $\mathrm{p}$ inferiores a $\mathrm{o,2}$, lo cual indica una asociación estadísticamente significativa, por lo que fueron incluidas en el modelo de regresión inicial (tabla 3).

Al elaborar el modelo de predicción con la cohorte de derivación para el punto compuesto de complicación y aplicar la estrategia backward para la selección de las variables, se encontró que las variables edad $(\mathrm{p}=0,004)$, diabetes mellitus $(\mathrm{p}=\mathrm{o}, \mathrm{O} 23)$, enfermedad renal crónica $(\mathrm{p}=\mathrm{o}, \mathrm{OI} 4)$, coledocolitiasis $(\mathrm{p}=\mathrm{O}, 004)$ y síndrome de Mirizzi ( $\mathrm{p}=\mathrm{o}, \mathrm{OOI}$ ) fueron significativas en el modelo final, lo que significa que son factores asociados con la presencia de complicaciones (tabla 4).

Al validar el modelo, se encontró que no existe colinealidad múltiple entre las variables (VIF<IO), y que éste presenta un buen ajuste. El modelo fue probado en la cohorte de validación, obteniéndose una capacidad de correcta clasificación del 58,27 \% (figura I).

\section{Discusión}

La colecistectomía por laparoscopia es el procedimiento de elección para el tratamiento de la colelitiasis y la colecistitis aguda, considerando la 
Tabla 3. Características de eventos con complicaciones y conversión ( $n=1230)$

\begin{tabular}{lccccccc}
\hline & $\begin{array}{c}\text { Conversión a } \\
\text { laparotomía } \\
\mathbf{n ~ ( \% )}\end{array}$ & $\begin{array}{c}\text { Lesión de } \\
\text { vía biliar* } \\
\mathbf{n}(\%)\end{array}$ & $\begin{array}{c}\text { Sangrado } \\
\mathbf{n}(\%)\end{array}$ & $\begin{array}{c}\text { Lesión } \\
\text { intestinal } \\
\mathbf{n}(\%)\end{array}$ & $\begin{array}{c}\text { Lesión } \\
\text { vascular } \\
\mathbf{n}(\%)\end{array}$ & $\begin{array}{c}\text { Colección } \\
\text { intra- } \\
\text { abdominal } \\
\mathbf{n}(\%)\end{array}$ & $\begin{array}{c}\text { Mortalidad } \\
\mathbf{n}(\%)\end{array}$ \\
\hline Total de eventos & $53(4,3)$ & $13(1,1)$ & $13(1,1)$ & $5(0,4)$ & $1(0,1)$ & $32(2,6)$ & $4(0,3)$ \\
Edad (años) mediana (RIQ) & $59(50-69)$ & $55(34-63)$ & $63(48-68)$ & $58(57-66)$ & $60(60-60)$ & $57,5(44,5-67,5)$ & $62,5(53,5-73)$ \\
Sexo: mujeres & $27(51,0)$ & $7(53,4)$ & $3(23,1)$ & $1(20,0)$ & 0 & $18(56,3)$ & $2(50,0)$ \\
Cirugía urgente & $48(90,6)$ & $12(92,3)$ & $11(84,6)$ & $2(40,0)$ & $1(100)$ & $22(68,8)$ & $3(75,0)$ \\
CPRE preoperatoria & $8(15,1)$ & $4(30,8)$ & $2(15,4)$ & $1(20,0)$ & 0 & $9(28,1)$ & $1(25,0)$ \\
Inserción abierta del trocar & $49(92,5)$ & $11(84,6)$ & $11(84,6)$ & $4(80,0)$ & 0 & $25(78,1)$ & $2(50,0)$ \\
Hallazgos quirúrgicos & & & & & & 0 & 0 \\
Hidrocolecisto & $1(1,9)$ & $1(7,7)$ & 0 & 0 & 0 & 0 & $7(21,9)$ \\
Piocolecisto & $22(41,5)$ & $3(23,1)$ & $2(15,4)$ & $2(40,0)$ & 0 & $2(50,0)$ \\
Plastrón vesicular & $32(60,4)$ & $4(30,8)$ & $4(30,8)$ & $2(40,0)$ & 0 & $7(21,9)$ & $2(50,0)$ \\
Colecistitis & $41(77,4)$ & $8(61,5)$ & $7(53,9)$ & $2(40,0)$ & 0 & $18(56,3)$ & $3(75,0)$ \\
Síndrome de Mirizzi & $15(28,3)$ & $2(15,4)$ & $2(15,4)$ & 0 & 0 & $3(9,4)$ & 0 \\
\hline
\end{tabular}

* Solo una lesión de vía biliar compleja con necesidad de reconstrucción

RIQ: rango intercuartílico ; CPRE: colangiopancreatografía retrógrada endoscópica

Tabla 4. Variables significativas del modelo de predicción en cohorte de derivación

\begin{tabular}{lcccccc}
\hline Resultado & Coeficiente & Error estándar & $\mathbf{z}$ & $\mathbf{P}>|\mathbf{z}|$ & \multicolumn{2}{c}{ [IC95\%] } \\
\hline Edad & 0,0318999 & 0,0112104 & 2,85 & 0,004 & 0,0099279 & 0,0538719 \\
Diabetes mellitus & 0,9684463 & 0,4245385 & 2,28 & 0,023 & 0,1363661 & 1,800526 \\
Enfermedad renal crónica & 1,698829 & 0,6936508 & 2,45 & 0,014 & 0,3392986 & 3,05836 \\
Coledocolitiasis & 1,199665 & 0,4112331 & 2,92 & 0,004 & 0,3936625 & 2,005667 \\
Síndrome de Mirizzi & 1,713497 & 0,5201019 & 3,29 & 0,001 & 0,6941161 & 2,732878 \\
Consolidado & $-5,279473$ & 0,6577235 & $-8,03$ & 0,000 & $-6,568587$ & $-3,990358$ \\
\hline
\end{tabular}

Número de observaciones para la regresión logística $=92 \mathrm{I}$

$\operatorname{LRX}^{2}(5)=38,70$

Probabilidad $>\mathrm{X}^{2}=0,0000$

Log likelihood = -I45,2302 Pseudo $\mathrm{R}^{2}=$ 0,II76

colecistitis como la complicación más frecuente de la colelitiasis ${ }^{17}$. Al ser un procedimiento tan frecuente, se han identificado claros factores de riesgo que podrían estar asociados con complicaciones propias de la cirugía, como lesión de la vía biliar, sangrado, colecciones intraabdominales, muerte ${ }^{18-20}$ o conversión a cirugía abierta ${ }^{21}$.

En concordancia con los datos encontrados en la literatura científica actual, que registran una frecuencia de colelitiasis mayor en el sexo femenino, en el presente estudio se registró un porcentaje de mujeres con colelitiasis de $69,2 \%{ }^{22}$.

Tratándose de un estudio llevado a cabo en una institución con alto nivel de complejidad que recibe pacientes que requieren manejos interdisciplinarios especializados, incluyendo especialidades médicas no disponibles en otras instituciones de menor nivel, y sabiendo que las enfermedades crónicas como la diabetes mellitus, hacen parte de los factores de riesgo para desarrollar colecistitis, se tuvo en cuenta la diabe- 


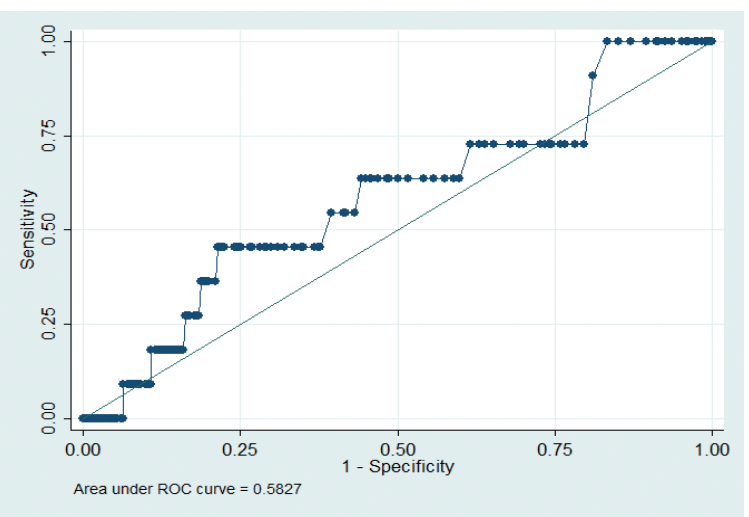

Figura 1. Curva ROC para el modelo predictivo, punto compuesto de complicación

tes mellitus como una variable estadísticamente significativa de predicción, ya que el $7,8 \%$ de la población de este estudio presentaba el antecedente de esta enfermedad.

Haciendo parte de los predictores de complicaciones de colecistectomía por laparoscopia, se encuentra el antecedente quirúrgico abdominal en un alto porcentaje de pacientes $(44,2 \%)$. Este antecedente está ligado a mayores dificultades técnicas, tanto para el ingreso de los trocares como para el procedimiento mismo, dada la presencia de adherencias en la cavidad abdominal; asimismo, las alteraciones de las pruebas de función hepática y del diámetro de la vía biliar común, hacen parte de estos factores predictores de coledocolitiasis, por lo que estos estudios se practicaron en la mayoría de los pacientes involucrados, encontrándose dilatación del colédoco en el II, $2 \%$.

De acuerdo con los datos estadísticos de incidencia de coledocoliatiasis en la población con colecistitis y teniendo en cuenta los predictores de coledocolitiasis ${ }^{23}$, se indicó la colangiopancreatografía retrógrada endoscópica en II,6 \% de los pacientes ${ }^{24}$. Asimismo, debido a que el resultado podría afectarse estadísticamente con el modelo de diagnóstico de coledocolitiasis, al igual que con la colangiopancreatografía retrógrada endoscópica, y debido a que clínicamente son variables complementarias, se decidió excluir este procedimiento como punto predictor de una complicación mayor y se analizó junto con la presencia de un diagnóstico de coledocolitiasis previo a la cirugía. Se tiende a practicar la colecistectomía en forma tardía con el fin de controlar el proceso inflamatorio y, así, disminuir las posibles complicaciones ${ }^{25}$.

La población estudiada en esta investigación se caracterizó según el tipo de cirugía, bien fuera por laparotomía o laparoscopia, y se tuvo en cuenta el tiempo transcurrido desde el momento del diagnóstico hasta el traslado a salas de cirugía para la realización de procedimiento, es decir, se tuvieron en cuenta aquellos con indicación de cirugía urgente y los que tenían opción de cirugía programada; además, se tuvieron en cuenta los hallazgos operatorios y la estancia hospitalaria. Los pacientes que requirieron cirugía urgente, se intervinieron en las primeras 72 horas de la hospitalización $(71,3 \%)$ de manera que, tal como se ha demostrado en la literatura, este fue un factor protector para disminuir la lesión de vía biliar y la tasa de conversión, y contribuyó a una estancia hospitalaria menor; en este estudio, esta presenta una mediana de dos días, cercana a la reportada en diferentes estudios, de I,9 días ${ }^{26}$.

La complejidad de los pacientes incluidos en el estudio, y el hecho de que el mayor porcentaje requirió cirugía de urgencia, condujo a tener como procedimiento convencional la colecistectomía por laparoscopia con cuatro puertos ${ }^{27}$. Entre los hallazgos operatorios, el más frecuente en cirugía por colelitiasis, urgente o programada, fue la colecistitis, presente en $43,5 \%$ de las intervenciones; el síndrome de Mirizzi, como factor dentro del modelo aquí estudiado, alcanzó el $3,5 \%$, valor equiparable con las cifras reportadas en la literatura científica, de 0,05 a $4 \%$ según la serie $^{20,28,29}$.

Cuando se inició el uso de la cirugía laparoscópica para la colelitiasis, se consideraba la inflamación aguda como una contraindicación para el abordaje laparoscópico debido a las tasas de conversión a laparotomía de hasta el $60 \%{ }^{(30)}$. Con la formalización del entrenamiento en cirugía laparoscópica, este abordaje se convirtió 
en el preferido, dejando a un lado la consideración de la conversión a técnica abierta como una complicación, aunque sin desconocer el impacto que este abordaje puede tener en la evolución clínica posoperatoria, en el manejo del dolor y en la estancia hospitalaria.

En la actualidad, los factores preoperatorios, como edad, colecistitis aguda e, incluso, el incremento de los leucocitos " ${ }^{\text {II }}$ las amilasas y otros marcadores bioquímicos (como las transaminasas y la bilirrubina), se relacionan con el proceso de conversión a técnica abierta. No obstante, consideramos que su principal causa es la imposibilidad de identificar apropiadamente las estructuras anatómicas durante el procedimiento ${ }^{6}$. Esta dificultad se reporta asociada con un porcentaje bajo (o a $20 \%$ ) de conversión, lo cual concuerda con su baja tasa en el presente estudio (4,3\%), menor que la informada en la mayoría de los estudios citados: 7,9\% para cirugía urgente y $4 \%$ para cirugía programada. Este factor permite, además, demostrar disminución en la estancia hospitalaria, el dolor posoperatorio y los costos de la atención ${ }^{\mathrm{II}, 30}$.

Teniendo en cuenta que las lesiones de vía biliar abarcan desde aquellas simples con fuga del muñón cístico, que pueden requerir un manejo expectante, hasta las complejas que obligan a reconstruir la vía biliar ${ }^{31}$, se calcularon las tasas de este tipo de complicaciones comparándolas con la tasa global reportada en la literatura, de hasta $0,7 \%{ }^{19}$, porcentaje que incluye lesiones avanzadas según la clasificación de Strasberg. En el presente estudio se obtuvo un porcentaje muy por debajo de la tasa global para este tipo de lesiones, o,o8\%.

Para el análisis, se tuvieron en cuenta factores asociados con los resultados, como la experiencia del cirujano, la colecistitis aguda y las variaciones anatómicas ${ }^{19}$; en el presente estudio, estos representaron una tasa de I,I \%. Otros factores que influyen en las complicaciones y afectan los resultados, son el síndrome de Mirizzi, analizado en estudios previos, y aquellos no considerados en otros estudios, como la edad, la diabetes mellitus, la enfermedad renal crónica y la coledocolitiasis.
En un estudio llevado a cabo en Suecia, se reporta una tasa global de sangrado antes y después de la cirugía de hasta I,4 \% ${ }^{32}$, un poco mayor que el encontrado en este estudio, que fue de I,I \%,

La tasa global de perforación intestinal reportada es de $0,27 \%$ 33; la encontrada aquí fue de $0,4 \%$ y, aunque un poco más alta que la anterior, no mostró asociación estadística con otros factores y, por su baja incidencia, se decidió no incluirla en el análisis como resultado compuesto de complicación.

La incidencia de colección intraabdominal reportada es de hasta I,2 \% de las colecistectomías por laparoscopia ${ }^{30}$; en nuestro medio, su proporción es mayor (2,6 \%). Esto puede estar asociado con las comorbilidades preoperatorias, como diabetes mellitus y enfermedad renal crónica, con una mayor edad y con el diagnóstico de coledocolitiasis y síndrome de Mirizzi. Estas dos últimas condiciones se relacionan con procesos agudos como la colangitis, que aumenta clínicamente el riesgo de colección intraabdominal, demostrado estadísticamente en este estudio en el punto compuesto de complicación. Al analizar esta complicación en forma aislada, se encuentra que la coledocolitiasis y la diabetes son los predictores más relevantes.

En este estudio se presentaron cuatro fallecimientos $(0,3 \%)$, solo uno de ellos relacionado con el procedimiento quirúrgico (sangrado); los tres restantes fueron ocasionados por complicaciones médicas. Este resultado es lo esperado si se compara con otros estudios similares, como uno llevado a cabo en Suecia, con mortalidad de 0, I a $0,7 \%{ }^{18}$.

Este estudio fue realizado en un centro de referencia, por lo que recibe casos de mayor complejidad y muy heterogéneos; sin embargo, la mayoría de los hallazgos se correlacionan con lo informado en la literatura científica.

El antecedente quirúrgico abdominal se presentó en un alto porcentaje $(44,2 \%)$, lo cual podría asociarse con mayor dificultad técnica para introducir los trocares, así como para el procedimiento mismo; sin embargo, no hubo diferencias estadísticamente significativas entre los indivi- 
duos con antecedente quirúrgico abdominal y los que no tenían este antecedente, probablemente por la mejoría de la curva de aprendizaje de este procedimiento ${ }^{34}$.

Aunque la conversión a cirugía abierta no se considera una complicación, es importante destacar que algunos factores pueden estar asociados con su mayor probabilidad, como el piocolecisto; aquí se encontró que el 19,6 \% de los pacientes con este diagnóstico requirieron conversión a laparotomía.

La única complicación que fue más frecuente en la cirugía programada, fue la lesión intestinal ( 3 de 5 casos). Sin embargo, en el conjunto de cirugías urgentes y programadas, la tasa global de lesión intestinal fue de $0,4 \%$, comparable con las de otras series similares, que alcanzan el o,2I \% ${ }^{35}$.

No se pudieron medir variables de interés como el riesgo anestésico y el índice de masa corporal, ni establecer si el procedimiento fue practicado por residentes en entrenamiento bajo supervisión de un cirujano tratante o por un cirujano tratante con ayuda de residentes en entrenamiento, lo cual podría impactar en resultados como conversión a cirugía abierta, tiempo quirúrgico o lesión de vía biliar. Esto se debió a la limitación en la recolección de información, la cual se obtuvo de las historias clínicas. Sin embargo, son de resaltar el número de pacientes incluidos, el número de variables analizadas y el seguimiento para establecer complicaciones perioperatorias.

\section{Conclusiones}

Con los datos analizados, se puede afirmar que factores como la edad, la diabetes mellitus, la enfermedad renal crónica, la coledocolitiasis y el hallazgo quirúrgico de síndrome de Mirizzi, influyen en el resultado de la colecistectomía por laparoscopia en adultos con colelitiasis por mayor probabilidad de complicación.

En este estudio se brinda información de un modelo predictivo como herramienta estadística para los cirujanos generales, con variables de fácil medición clínica y de bajo costo, que permite conocer la probabilidad de complicacio- nes mayores, como colección abdominal, lesión de la vía biliar o sangrado en la colecistectomía por laparoscopia. Con su uso, se pueden generar estrategias de prevención, identificando aquelos pacientes que presenten baja tolerancia a la conversión y riesgo de complicaciones en la colecistectomía por laparoscopia, en cuyo caso el procedimiento deberá ser realizado por cirujanos con amplia experiencia y contar con reserva de hemoderivados, entre otras.

Este estudio puede servir de base para estudios posteriores que busquen validar el modelo de predicción de complicaciones, dando pie a futuras investigaciones de interés para el cirujano en general.

\section{Agradecimientos}

Al Servicio de Cirugía General del Hospital de San José y a la División de Investigaciones de la Fundación Universitaria de Ciencias de la Salud.

\section{Declaración de conflicto de intereses}

Los autores declaran no tener conflicto de intereses.

\section{Declaración de financiación del proyecto}

El proyecto no recibió financiación.

\section{Referencias}

I. O'Connell K, Brasel K. Bile metabolism and lithogenesis. Surg Clin North Am. 20I4;94:36I-75.

2. Knab LM, Boller AM, Mahvi DM. Cholecystitis. Surg Clin North Am. 20I4;94:455-70.

3. Gaitán JA, Martínez VM. Enfermedad litiásica biliar, experiencia en una clínica de cuarto nivel, 2005-20II. Rev Colomb Cir. 20I4;29:I88-96.

4. Lambou-Gianoukos S, Heller SJ. Lithogenesis and bile metabolism. Surg Clin North Am. 2008;88:II75-94.

5. Fletcher DR, Hobbs MS, Tan P, Valinsky LJ, Hockey RL, Pikora TJ, et al. Complications of cholecystectomy: Risks of the laparoscopic approach and protective effects of operative cholangiography: A population-based study. Ann Surg. 1999;229:449-57.

6. Álvarez LF, Rivera D, Esmeral ME, García MC, Toro DF, Rojas OL. Colecistectomía laparoscópica difícil, estrategias de manejo. Rev Colomb Cir. 2013;28:186-95.

7. Strasberg SM. Clinical practice. Acute calculous cholecystitis. N Engl J Med. 2008;358:2804-II.

8. Russell JC, Walsh SJ, Reed-Fourquet L, Mattie A, Lynch J. Symptomatic cholelithiasis: A different disease in 
men? Connecticut Laparoscopic Cholesystectomy Registry. Ann Surg. 1998;227:195-200.

9. Takada T, Strasberg SM, Solomkin JS, Pitt HA, Gomi H, Yoshida M, et al. TGı3: Updated Tokyo Guidelines for the management of acute cholangitis and cholecystitis. J Hepatobiliary Pancreat Sci. 2013;20:I-7.

Io. Ruiz C, Cazares J, Muñiz M, García A. Dificultados técnicas en la colecistectomía laparoscópica. La "colecistectomía difícil». Rev Mex Cir Endoscop. 20I0;II:84-93.

II. Licciardello A, Arena M, Nicosia A, Di Stefano B, Calì G, Arena G, et al. Preoperative risk factors for conversion from laparoscopic to open cholecystectomy. Eur Rev Med Pharmacol Sci. 20I4;18(Suppl.):60-8.

I2. Wevers KP, van Westreenen HL, Patijn GA. Laparoscopic cholecystectomy in acute cholecystitis: C-reactive protein level combined with age predicts conversion. Surg Laparosc Endosc Percutan Tech. 2013;23:163-6.

13. Le VH, Smith DE, Johnson BL. Conversion of laparoscopic to open cholecystectomy in the current era of laparoscopic surgery. Am Surg. 2012;78:1392-5.

I4. Overby DW, Apelgren KN, Richardson W, Fanelli R, Surgeons SoAGaE. SAGES guidelines for the clinical application of laparoscopic biliary tract surgery. Surg Endosc. 20I0;24:2368-86.

I5. Murphy MM, Ng SC, Simons JP, Csikesz NG, Shah SA, Tseng JF. Predictors of major complications after laparoscopic cholecystectomy: surgeon, hospital, or patient? J Am Coll Surg. 20I0;2II:73-80.

I6. Stanisic V, Milicevic M, Kocev N, Stojanovic M, Vlaovic $\mathrm{D}$, Babic I, et al. Prediction of difficulties in laparoscopic cholecystectomy on the base of routinely available parameters in a smaller regional hospital. Eur Rev Med Pharmacol Sci. 20I4;18:I2O4-II.

17. Koti RS, Davidson CJ, Davidson BR. Surgical management of acute cholecystitis. Langenbecks Arch Surg. 2015;400:403-19.

I8. Sandblom G, Videhult P, Crona Guterstam Y, Svenner A, Sadr-Azodi O. Mortality after a cholecystectomy: A population-based study. HPB (Oxford). 2015;17:239-43.

19. Fortunato AA, Gentile JK, Caetano DP, Gomes MA, Bassi MA. Comparative analysis of iatrogenic injury of biliary tract in laparotomic and laparoscopic cholecystectomy. Arq Bras Cir Dig. 20I4;27:272-4.

20. Díaz S, Correa MJ, Giraldo LM, Ríos DC, Solórzano F, Wolff JD, et al. Experiencia en colecistectomía por laparoscopia en la Clínica Universitaria CES. Rev Colomb Cir. 2012;27:275-80.

2I. Sugrue M, Sahebally SM, Ansaloni L, Zielinski MD. Grading operative findings at laparoscopic cholecystectomy- a new scoring system. World J Emerg Surg. 20I5;IO:I4.
22. Abraham S, Rivero HG, Erlikh IV, Griffith LF, Kondamudi VK. Surgical and nonsurgical management of gallstones. Am Fam Physician. 2014;89:795-802.

23. Bejarano M. Utilidad de los factores predictores de coledocolitiasis en pacientes operados en la Clínica Rafael Uribe Uribe - Cali. Rev Colomb Cir. 2003;18:73-83.

24. Bencini L, Tommasi C, Manetti R, Farsi M. Modern approach to cholecysto-choledocholithiasis. World J Gastrointest Endosc. 20I4;6:32-40.

25. Ciftci F, Abdurrahman I, Girgin S. The outcome of early laparoscopic surgery to treat acute cholecystitis: A single-center experience. Int J Clin Exp Med. 2015;8:4563-8.

26. de Mestral C, Rotstein OD, Laupacis A, Hoch JS, Zagorski B, Alali AS, et al. Comparative operative outcomes of early and delayed cholecystectomy for acute cholecystitis: a population-based propensity score analysis. Ann Surg. 2014;259:10-5.

27. Ahmed MU, Aftab A, Seriwala HM, Khan AM, Anis K, Ahmed I, et al. Can single incision laproscopic cholecystectomy replace the traditional four port laproscopic approach: A review. Glob J Health Sci. 20I4;6:II9-25.

28. Waisberg J, Corona A, de Abreu IW, Farah JF, Lupinacci RA, Goffi FS. Benign obstruction of the common hepatic duct (Mirizzi syndrome): Diagnosis and operative management. Arq Gastroenterol. 2005;42:13-8.

29. Beltrán MA. Mirizzi syndrome: History, current knowledge and proposal of a simplified classification. World J Gastroenterol. 2012;18:4639-50.

30. Oymaci E, Ucar AD, Aydogan S, Sari E, Erkan N, Yildirim $\mathrm{M}$. Evaluation of affecting factors for conversion to open cholecystectomy in acute cholecystitis. Prz Gastroenterol. 20I4;9:336-4I.

3I. Barbier L, Souche R, Slim K, Ah-Soune P. Long-term consequences of bile duct injury after cholecystectomy. J Visc Surg. 20I4;15I:269-79.

32. Persson G, Strömberg J, Svennblad B, Sandblom G. Risk of bleeding associated with use of systemic thromboembolic prophylaxis during laparoscopic cholecystectomy. Br J Surg. 20I2;99:979-86.

33. Nijssen MA, Schreinemakers JM, Meyer Z, van der Schelling GP, Crolla RM, Rijken AM. Complications After laparoscopic cholecystectomy: A video evaluation study of whether the critical view of safety was reached. World J Surg. 2015;39:1798-803.

34. Liu CM, Hsu CT, Li CY, Chen CC, Liu ML, Liu JH. A population-based cohort study of symptomatic gallstone disease in diabetic patients. World J Gastroenterol. 2012;18:1652-9.

35. Andrews S. Does concentration of surgical expertise improve outcomes for laparoscopic cholecystectomy? 9 year audit cycle. Surgeon. 2013;II:309-I2. 